ständigen Epispadie zu sein, in welcher Beziehung der erstere Fall auch nicht ohne Interesse war, indem es nachgewiesen wurde, dass dem Paienten trotz seiner mannigfachen Verbindungen niemals die Vaterschaft eines unehelicben. Kindes zugeschoben worden war.

\title{
XVII.
}

\section{Das zweite Stadium der Alopecia pityrodes.}

\author{
Von Stabsarzt Dr. Pincus in Diez.
}

Das erste Stadium der Alopecie charakterisirt sich ${ }^{*}$ ) wesentlich durch eine allmähliche Abnahme des typischen Längenwachstbums des einzelnen Kopfhaares, verbunden mit einer über den ganzen Kopf ausgebreiteten, am Mittelkopfe besonders stark hervortretenden Pityriasis.

Das charakteristische Moment des zweiten Stadiums ist eine Abnahme des Dickendurchmessers des einzelnen Haares.

Schon eine oberflächliche Betrachtung lehrt, dass bei völlig gesundem Haarwuchs der Dickendurchmesser der einzelnen Haare in ein und derselben Gegend variirt. Prüft man bei kurzgeschorenem Haar kleine Haarkreise an Stücken einer Kopfhaut, die in absolutem Alkohol gehärtet ist, so findet man regelmässig wiederkehrende Variationen, so regelmässig, dass der typische Charakter dieser Variationen nicht zu verkennen ist. Die Haare stehen zu je 3, 4 oder 5 zusammen; Schnitte, parallel der Oberfläche, zeigen (besonders in den oberen Schichten der Cutis) eine den einzeinen Haarkreisen entsprechende Anordnung des Fasergerüstes der Haut: jeder Haarkreis wird gebildet von einem verbältnissmässig breiten Faserbande, von welchem erbeblich schwächere Züge nach dem Innern des Haarkreises abgehen, um ihrerseits wiederum kleinere Ringe für die einzelnen Haarkreise zu bilden.

Bei Menschen von kräftigem Haarwuchs ist die Differenz des Dickendurehmessers der einzelnen, zu einem Kreise gehörenden

*) Bd. XXXVII dieses Archivs S. 29. 
Haare sehr gering; dass ein Haar mehr als das Doppelte des Dikkendurchmessers seiner Nachbaril bat, ist die Ausnahme; eine Anordnung, entsprechend der bei einzelnen Thieren vorkommenden Anordnung der Stichelhaare habe ich nie gesehen. Bei der Beurtheilung muss man nur von denjenigen einzelnen Kreisen abstrahiren, innerhalb deren ein ganz junges Haar sich befindet, weil die Haarspitze auch bei dem kräftigsten Haarwuchs einen erheblich geringeren Durchmesser hat als der später nachwachsende Stamm. Der Dickendurchmesser des stärksten Haares eines Haarkreises verhält sich zu dem der anderen Kreisgenossen etwa wie 5 zu 4, selten ist das Uebergewicht grösser.

Der Durchmesser des Hautgebietes, welches abgegrenzt durch die oben erwähnten schmalen, den Haarbalg umschliessenden Faserzüge zu jedem Haar gehört, variirt mit dem Dickendurchmesser des Haares and steigt mit demselben; hingegen habe ich eine wesentlich verschiedene Breite des begrenzenden Faserzuges selbst nicht gefunden.

Am Kopfe des Lebenden lassen sich bei nicht zu dichtem Haarwuchs die einzelnen Harkreise erkennen, besonders wenn der Dickendurchmesser der zu einem Kreise gehörenden Haare nicht zu unbedeutend variirt; am deutlichsten auf der Höhe des Scheitels und in der Nähe der Stirn, scbwerer an den Schläfen und am Hinterkopfe. Es ist jedoch nicht möglich, am normalen Kopfhaar eines Lebenden die verschiedenen Wacbsthumsverhältnisse der einzelnen Haare eines Kreises zu beobachten: die beträchtliche typische Länge dieser Haare und die in der Regel mehr als 2 bis 3 jährige Lebensdauer machen es unmöglich, den Entwicklungsgang des neuen Haares dauernd zu verfolgen.

Wesentlich günstiger für die Beobachtung liegen die Verhältnisse bei den Haaren an der Dorsalfläche der Finger: Die zu einem Haarkreis gehörenden Haare stehen fast eben so dicht wie am Kopf, aber der freie Raum zwischen den Kreisen ist erheblich grösser; das typische Längenwachsthum des Haares beträgt 2 bis 9 Linien; die typische Lebensdauer 3 bis 9 Monat: es ist bei einiger Uebung leicht möglich, den Lebensprozess eines ganzen Kreises zu verfolgen, besonders da der Kreis gewöhnlich nur 3 oder 4 Haare enthält.

Zur Beobachtung wählt man am Besten einen Kreis, in welchem vor Kurzem erst ein Haar ausgefallen ist, oder einen solchen, 
innerhalb dessen man ein junges Härchen schon hervorspriessen sieht. Man findet in diesem Kreise alsdann, wenn er 4 Haare umfasst, gewöhnlich 2 Haare von ungefïhr gleicher Länge und ein viertes erbeblich länger oder kürzer als die beiden anderen.

Die Beobachtung lehrt nun zweierlei:

1) Die Lebensdauer, die typische Länge und der Dickendurchmesser der 4 Ha are variiren untereinander nicht unbeträchtlich. Das dickste Haar hat die längste Lebensdauer und erreicht die grösste Länge, ohne dass jedoch eine erhebliche Differenz in der Geschwindigkeït des Wachsthums des dickeren und der dïnnejen Haare sich nachweisen lässt. Die Lebensdauer des dicken Haares betrug in den von mir beobachteten Fällen 7 bis 9 Monat, die der dünneren 3 bis 5 Monat; in gleichem Verhältniss etwa standen die bezüglichen Dickendurchmesser.

Diese Zahlenverhältnisse sind für die verschiedenen Finger ein und desselben Individuums wenig variirend; ich vermuthe indessen, dass sie bei verschiedenen Individuen beträchtlich variiren.

2) Der Zeitpunkt des Lebensendes fällt für die einzelnen Haare des Kreises nie zusammen; es vergeht in der Regel wenigstens ein Viertel der Lebensdauer der kürzeren Haare (also etwa ein Zeitraum von 3 bis 5 Wochen), bevor dem früher ausgefallenen Haar ein zweites aus demselben Kreise folgt.

Dieses beim normalen Gesundheitszustande regelmässig wiederkehrende Verhältniss muss in typischen Wachsthumsverhältnissen seinen Grund haben: welcher Art dieselben sein mögen, weiss ich nicht zu sagen; nur das möchte ich fast als gewiss annehmen, dass die Blutcirculations - Verbältnisse keinen unmittelbaren Einfluss ausüben.

Selbstverständlich gilt dieses Gesetz nur für normale Verbältnisse; bei fieberhaften Krankheiten von längerer Dauer, besonders bei Exanthemen, ist ein sehr rasch auf einander folgendes Ausfallen mehrerer oder sämmtlicher Haare eines Kreises auch am Fingerricken oft zu constatiren. Ein wahres Defluvium indessen, entsprechend dem Defluvium capillorum, habe ich nie gesehen.

In der Mittheilung über das erste Stadium der Alopecie *) habe ich bereits erwähnt, dass die Frage, ob die Abnabme der 
typischen Länge des kranken Haares völlig gleichmässig erfolge oder nicht, sich dusch directe Beobachtung nicht entscheiden lasse. Es lässt sich auch für den Anfang des zweiten Stadiums der Alopecie nicht durch directe Beobachtung constatiren, ob die allmähliche Abuahme des Dickendurchmessers bei den einzelnen Haaren ein und desselben $\mathrm{Kr}$ eises gleichmässig erfolge oder nicht.

Bei weiterem Fortschreiten des zweiten Stadiums hingegen, d. h. zu dem Zeitpunkte, wenn bereits ein Lichtwerden des Haarbodens dem Auge bemerkbar geworden, lässt sich das bezügliche Verbältniss genau erkennen.

Es ist alsdann zunächst leicht festzustellen, dass die Haarkreise des Mittel- und Vorderkopfes nicht in gleicher Weise ergriffen werden. Das Leiden erscheint am meisten vorgeschritten an einem 1 bis $1 \frac{1}{2}$ Zoll breiten Streifen, der etwa $3 / 4$ Zoll hinter dem corderen Rande des Haarwuchses beginut; dann folgt die Höhe des Scheitels, dann der Rest des Mittelkopfes; der vorderste Rand des Haarwuchses, besonders in der Nähe der Mittellinie, widersteht dem Krankheitsprozess am längsten. Untersucht man nun die Stellen, an denen der Krankheitsprozess am meisten vorgeschritten ist, wiederholt und genau (am besten, indem man kleine Kreise mit einer Höllensteinauflösung zeichnet), so findet man innerhalb ein und desselben Haarkreises in der Regel 1 Haar mit normalem Dickendurchmesser, 1 oder 2 Haare von mittlerer Feinheit und 2 oder 1 von der Dünnheit des Wollhaars. Bei weiterem Fortschreiten des Leidens ist diess Verbältniss auf der Höhe des Scheitels, an welcher Stelle die einzelnen Haarkreise sich dann am leichtesten übersehen lassen, auch schon bei kiurzer dauernder Beobachtung nachzuweisen.

Bei weiterem Fortschreiten des Uebels ist an den erkrankten Stellen ein Haar von normaler Dicke nicht mehr zu finden, aber immer noch ist die Differenz der Dickendurchmesser der Haare ein und desselben Kreises sehr deutlich: neben 1 oder 2 mittelfeinen Haaren finden sich 2 bis 3 Wollhare; jene sind noch 1 bis 2 Zoll lang, diese 2 bis 5 Linien.

Allmählich wird auch das letzte Haar eines jeden Kreises in demselben Grade ergriffen, wie die übrigen Kreis-Einsassen: dann deckt kurzes, wenig gefärbtes Lanugo-Haar gleichmässig die Haut. Die ungleiche Widerstandskraft der einzelnen Haare ein und 


\section{6}

desselben Kreises kann entweder daher rühren, dass der Krankbeitsprozess sie nicht gleichzeitig mit derselben Intensität ergreift, oder daher, dass sie eine verschiedene ursprüngliche Kräftigkeit haben. Es ist schon oben erwähnt, dass bei den mejsten Menschen die Haare eines Kreises auch bei sehr kräftigem Haarwuchs nicht denselben Dickendurchmesser zeigen: die ursprünglich dünneren Haare werden dem Krankheitsprozesse eher erliegen. Allein man fjndet auch Fälle, in denen die gesunden Haare an der Schläfe und am Hinterkopf eine fast gleichmässige Dicke haben und bei denen dennoch auf dem erkrankten Mittelkopfe in den einzelnen Kreisen normal dicke Haare neben Lanngo stehen. Das Dickenverhältniss der einzelnen Haare ist aber für verschiedene Kreise desselben Kopfes ein und dasselbe, es lässt sich daher mit Sicherheit annehmen, dass auch in jenen Fällen auf dem Mittelkopte vor seiner Erkrankung Haare von ungefähr gleicher Dicke gestanden haben - es beweisen daber jene Fälle, dass es dem in Rede stebenden Krankheitsprozesse eigenthümlich ist, zwar vielfache Haarkreise zu gleicber Zeit und mit annähernd gleicher Intensität zu befallen, jedoch innerhalb jedes einzelnen Kreises eine regelmässige Differenzirung der Intensität zu zeigen.

Um den Entwickelungsgang des zweiten Stadiums übersichtlich darzustellen, will ich den an mir selbst wahrgenommenen Haarausfall mittheilen. Die Zablen hinter dem + bezeichnen die Spitzenhare, d. h. diejenigen Haare, welche eine so kurze Lebensdauer hatten, dass sie ausfielen, bevor sie die Länge von im Durchschnitt $4 \frac{1}{2}$ Zoll erreichten.

Dr. Pincus (1859), 25 Jahre alt, Haar hraunschwarz, mittelfein, selur dicht.

Kopflaut sehuppt sich seit 8 Jahren, seit 5 Jabren sebr bedeutend. Durchschnittslänge des Kopfhaares auf dem Mittelkopfe $4 \frac{1}{2}$ Zoll.

$$
\text { Erstes Stadium. }
$$

\begin{tabular}{|c|c|c|c|c|c|c|c|}
\hline \multicolumn{8}{|c|}{1859.} \\
\hline 25. Juni & $84+21$ & 3. Juli & $60+14$ & 5. Sept. & $68+14$ & 6. Nov. & $58+1$ \\
\hline $26 . \quad-$ & $87+25$ & $4 .-$ & $54+11$ & $6 .-$ & $64+13$ & $7 . \quad-$ & $64 t$ \\
\hline 27. & $76+16$ & 5. - & $51+9$ & 7. & $61+12$ & 8. & $68+1$ \\
\hline 28. & $73+15$ & 6. - & $55+14$ & 8. & $60+15$ & 9. & $60+1$ \\
\hline 29. & $75+-17$ & 1. Sept. & $54+14$ & 9. & $49+12$ & 10. & $70+1$ \\
\hline 30. & $69+15$ & $2 . \quad-$ & $56+10$ & 10. & $48+17$ & 11. & $56+1$ \\
\hline 1. Juli & $65+11$ & 3. & $57+14$ & 4. Nov. & $57+16$ & Minimum & 60 \\
\hline 2. & $60+8$ & & $50+18$ & 5. - & $50+10$ & Maximum & 112 \\
\hline & & & & & & Mittel & \\
\hline
\end{tabular}

Verbältniss der Spitzenhaare zum Gesammtausfall $1: 5,3533$. 
Zweites Stadium.

\begin{tabular}{|c|c|c|c|c|c|c|c|c|c|c|c|c|c|c|c|}
\hline \multirow{2}{*}{$\begin{array}{c}\text { Datum. } \\
1862 .\end{array}$} & \multicolumn{4}{|c|}{ 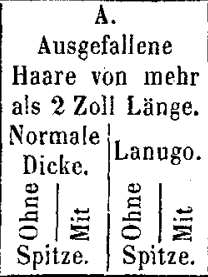 } & \multicolumn{4}{|c|}{ 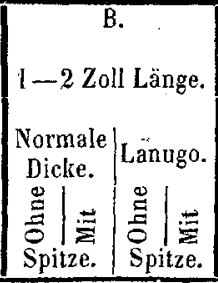 } & \multicolumn{4}{|c|}{ 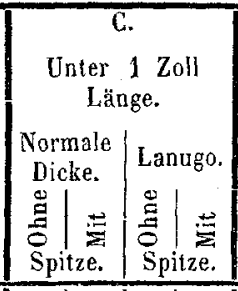 } & 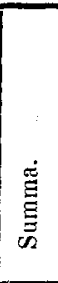 & \multirow[t]{2}{*}{$\begin{array}{c}\text { Zuschlag von } \\
\text { nicht gezählten } \\
\text { beim Waschen } \\
\text { efc. ausgefal- } \\
\text { lenen, nach } \\
\text { ungefährer } \\
\text { Schätzung. }\end{array}$} & 窎 \\
\hline & 22 & 8 & 3 & 4 & - & - & -1 & - & - & - & - & -1 & - & & 97 \\
\hline 18. & 25 & - & 3 & 2 & 25 & 10 & 2 & 1 & 26 & 30 & - & 16 & 140 & $B+C=25$ & 165 \\
\hline 19. & 9 & 9 & -1 & - & 9 & 3 & - & - & 8 & 8 & - & 9 & 55 & $20 \mathrm{C}, 10 \mathrm{~B}, 5 \mathrm{~A}$ & 90 \\
\hline 20. & 48 & 1 & - & - & 21 & 9 & - & 5 & 13 & 17 & - & 2 & 116 & $10 \mathrm{C}, 20 \mathrm{~B}, 10 \mathrm{~A}$ & 186 \\
\hline 1. & 26 & 5 & -1 & - & 8 & 8 & - & 3 & 16 & 11 & - & 25 & 102 & $5 \mathrm{~B}, 10 \mathrm{C}$ & 117 \\
\hline 2. & 37 & 6 & - & - & 24 & 24 & - & 6 & 51 & 43 & - & 28 & 219 & 90 & 309 \\
\hline 23 . & 17 & 2 & - & - & 12 & 7 & - & - & 16 & 14 & - & 11 & 79 & 一 & 79 \\
\hline 4. & 34 & 4 & -1 & - & 16 & 26 & - & 6 & 10 & 25 & $\ldots$ & 5 & 126 & 60 & 186 \\
\hline 25. & 42 & 8 & - & 2 & 39 & 28 & - & 4 & 59 & 62 & - & 9 & 255 & 15 & 270 \\
\hline 26 . & 30 & 2 & - & - & 18 & 22 & - & 1 & 28 & 16 & - & 23 & 140 & 10 & 100 \\
\hline 27. & 38 & 2 & - & - & 17 & 27 & - & -1 & 20 & 12 & - & - & 116 & 1 & 101 \\
\hline 28. & 45 & - & - & - & 48 & 30 & - & -1 & 40 & 34 & - & - & & 2 & 17 \\
\hline 29. & 16 & 7 & - & - & 18 & 19 & 1 & -1 & 25 & $1 \%$ & - & 10 & 113 & 20 & 33 \\
\hline 30. & 9 & 2 & - & - & $9\}$ & 7 & - & -1 & 17 & 3 & - & $\tilde{j}$ & 52 & & 62 \\
\hline 31. & 45 & 4 & -1 & - & 16 & 11 & -- & 1 & 26 & 12 & -.. & 14 & 109 & 15 & 24 \\
\hline 1. Aug. & 24 & 1 & - & - & 18 & 9 & - & 2 & 55 & 14 & 2 & 19 & 144 & 25 & 169 \\
\hline 2. & 13 & 4 & - & - & 11 & 9 & 1 & 1 & 32 & 8 & 4 & 4 & 7 & 10 & 87 \\
\hline 3. & 2 & 2 & $\check{5}$ & - & 31 & 8 & 1 & $i$ & 26 & 12 & 3 & 8 & 122 & $10 \mathrm{~B}, 10 \mathrm{C}$ & 142 \\
\hline 4. & 39 & 7 & - & - & 37 & 23 & 2 & 2 & 43 & 75 & 6 & 20 & 194 & 20 & 214 \\
\hline 5. & 39 & 3 & - & -1 & 29 & 15 & 2 & 2 & 32 & 15 & 2 & 14 & 253 & 1 & 168 \\
\hline 6. & 76 & 7 & - & - & 114 & 21 & & -1 & 149 & 65 & 4 & 6 & 442 & 4 & 482 \\
\hline 7. & 24 & 4 & - & - & 10 & 10 & -1 & - & 6 & 12 & - & -1 & 66 & 10 & 76 \\
\hline 8. & 34 & 5 & - & - & 6 & 11 & -1 & - & 8 & 7 & - & 一 & 71 & 4 & 101 \\
\hline 9. & 20 & 7 & - & -1 & 15 & 2 & - & -1 & 9 & 4 & - & - & 57 & 4 & 97 \\
\hline 10. & 51 & 13 & - & -1 & 39 & 15 & -1 & -1 & 23 & 17 & - & -1 & 158 & 20 & 178 \\
\hline 11. & 37 & 6 & - & - & 24 & 11 & - & 2 & 21 & 10 & 3 & 9 & 123 & 25 & \\
\hline 12. & 51 & 16 & - & - & 22 & 20 & - & - & 27 & $2 \tilde{5}$ & $-?$ & - & 161 & & \\
\hline 13. & 60 & 7 & - & - & 21 & 8 & 1 & 2 & 26 & 14 & 5 & 10 & 154 & 20 & 74 \\
\hline 14. & 55 & 13 & - & - & 31 & 15 & - & - & $30 !$ & 15 & - & - & & & 1 \\
\hline 15 & 56 ? & $\tilde{5}$ & - & - & $25 \mid$ & 19 & - & - & $40 \mid$ & 37 & - & - & 2 & 30 & \\
\hline 16 & 82 & 16 & - & - & 116 & 37 & - & -1 & 214 & 202 & -1 & - & 667 & 20 & 887 \\
\hline 17. & 36 & 2 & - & - & 26 & 20 & - & - & 56 & 62 & -1 & - & 2 & 18 & 120 \\
\hline 18 & 46 & 7 & - & - & 20 & 10 & - & - & 49 & 48 & - & - & 0 & 15 & 193 \\
\hline 19 & 66 & 8 & - & $-\ldots$ & 33 & 15 & $\cdots$ & - & 47 & 32 & -1 & - & I & 2 & \\
\hline 20 & 32 & 9 & - & - & 23 & 20 & - & - & 39 & 42 & -1 & - & 162 & 15 & 180 \\
\hline 21. & 48 & 9 & $1-$ & - & 27 & 34 & - & - & 62 & 72 & - & - & 252 & ? & \\
\hline 22 & 68 & 7 & - & - & 39 & 20 & - & - & 46 & 37 & -1 & - & 2 & ? & \\
\hline 23 & 41 & 3 & - & - & 47 & 19 & - & - & 60 & 34 & - & - & 2 & 15 & 219 \\
\hline 24 & 49 & 7 & - & - & 37 & 10 & - & - & 79 & 30 & - & - & 212 & $?$ & \\
\hline 25. & 76 & 12 & - & - & 41 & 25 & - & - & 123 & 79 & - & - & 3 & $?$ & \\
\hline 26 & 116 & 11 & - & - & 93 & 5 & 1 & 1 & 221 & 17 & 30 & 15 & 5 & $?$ & \\
\hline 27. & 28 & 1 & - & - & 26 & 4 & 3 & 3 & 28 & 15 & 28 & 25 & 161 & $?$ & \\
\hline 28. & 58 & 15 & $1-$ & $\ldots$ & 49 & 16 & $1-$ & $1-$ & 45 & 26 & 281 & 18 & 245 & $?$ & \\
\hline
\end{tabular}




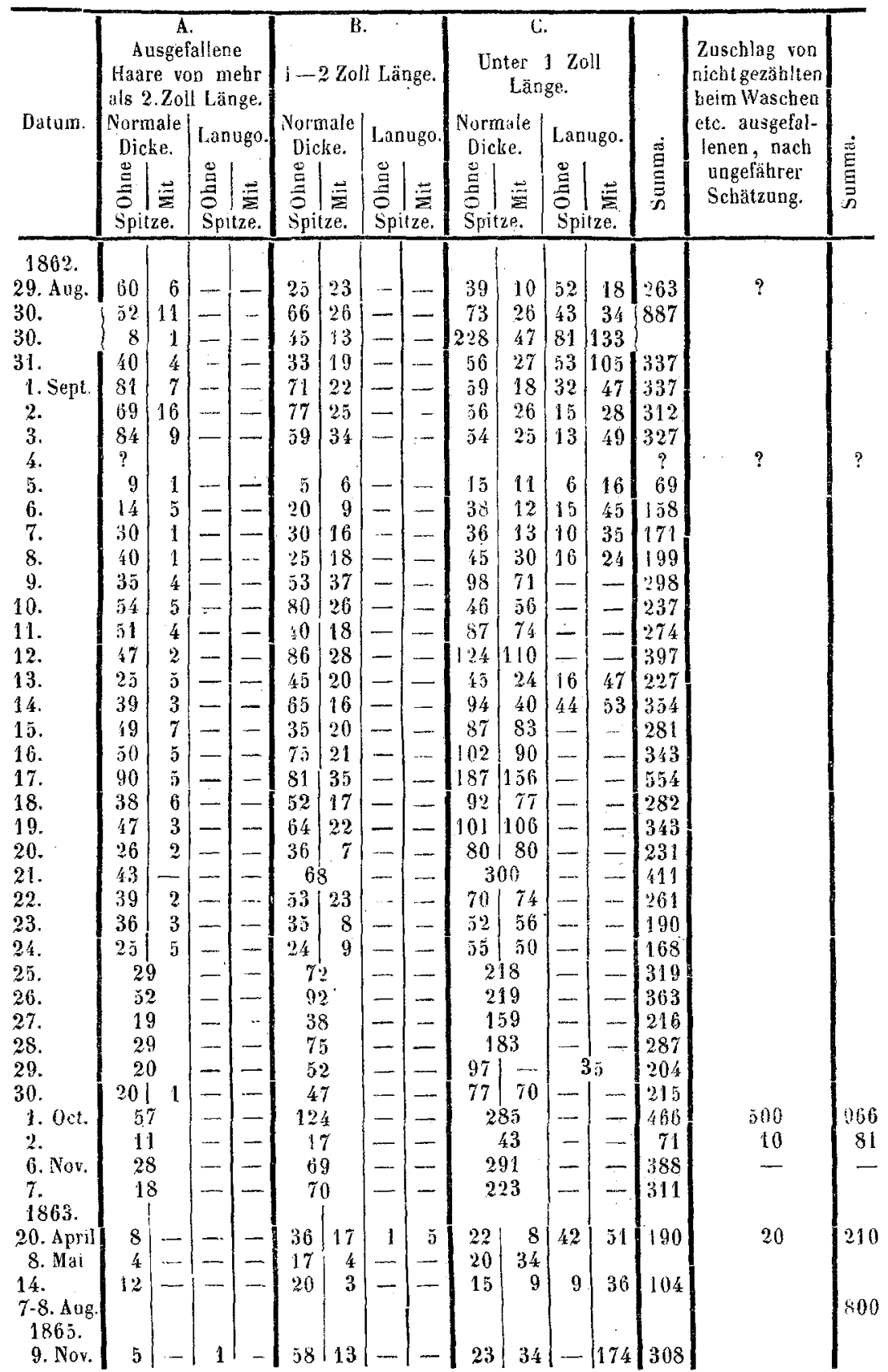


Die angeführte Zahlenreibe zeigt im Wesentlichen Folgendes:

Der absolute Haarausfall steigt mit dem Fortscbreiten des Leidens. Im ersten Stadium des Uebels betrug der tägiiche Veriust im Durchschnitt 76 . Er stieg im zweiten Stadium auf 135, dann auf 250 , allmählich auf 300 . Diese letzteren Zahlen gehen ausserordentlich ïber das normale Verhältniss hinaus: sie finden sich aber erst in einem Stadium der Krarkheit, in welchem es nicht der Zählung des täylichen Ausfalls, sondern nur eines Biickes auf den Korf bedarf, um den vorgeschritienen Grad des Leidens zu constatiren.

Auffallender noch als der absolute Haarausfall ist das abnorme Verhältniss der Spitzenhaare zum Gesamıtausfall. Dasselbe betrug im ersten Stadium 1 zu 5,353. Es stieg im zweilen Stadium auf $1 \mathrm{zu} 2,4$.

Diess Verbältniss zwischen den normallebigen und den kurzlebigen Haaren wird erst in das rechte Licht gestellt, wenn man nur den Ausfall des baarkranken Mittelkopfes in Betracht zieht, was durch sorgfältiges. Schlichten mit dem Staubkamm möglich ist. Ich habe solche Zählungen nur an meinem eigenen Haarausfall vorgenommen. Es fielen aus bei einer Zählung von 8 Tagen durchschnitllich pro Tag von den gesunden Kopftheilen 108, von den erkrankten 227, (es war Hocbsowmer und der absolute Haarausfall höher als sonst im Durchschnitt). Die enkrankte Kopfstelle halte etwa die Hälfte des Flächenraums der gesunden, dennoch lieferte sie täglich mehr als den doppelten Ausfall der gesunden, d. h. also: die Lebensdauer des einzelnen Haares an der erkrankten Stelle betrug durchschnitlich nur den vierten Theil derjenigen Zeit, weiche die übrigen Hare hatten. Dabei war das Verhältniss der Haare mit Spitze zu denen ohne Spitze für die gesunden Kopftheile $1 \mathrm{zu} 4$, für die kranken $1 \mathrm{zu} 1$ (das grosse Contingent der Spitzenhare auch für die scheinbar gesunden Kopfpartien wird nach dem im früheren Aufsatze über dieses Zahlenverhältniss Erwähnten nicht auffallen).

Die Iritensität des Erkrankungsprozesses zeigt sich für das zweite Stadium (dentlicher noch als durch die Steigerung des absoluten Haarausfalls und des Antheils der Spitzenhaare an demselben) durch die Zunahme der ausfallenden Lanugo-Haare. Ich war Anfangs der Ansicht, dass der Dickendurchmesser des früheren 
normalen Haares bis zu diesem Minimum erst sehr spät herabsinkt, etwa wenn die typische Länge bereits bis auf einen Zoll herabgegangen ist. Eine genaue Sichtung des Haarausfalles erwies jedoch diese Ansicht als eine irrige. Allerdings tritt der Lanugo-Charakter in der Regel erst bei einer typischen Länge des Haares von einem Zoll und darunter ein, allein man findet auch, etwa im zweiten oder dritten Jahre nach dem Beginne des zweiten Stadiums, Lanugo-Haare von 6 Zoll und darüber. In der Regel stammen allerdings diese längeren Lanugo-Haare (wie auch die oben üher meinen Haarausfall angegebenen Zahlen zeigen) nicht von dem erkrankten Mittelkopf, sondern von den übrigen Kopfpartien, besonders von den Schläfen (sie sind die am leichtesten aufzufindenden Beweismomente für die in dem ersten Aulsalz ausgesprochene Ansicht, dass bei der in Rede stebenden Alopecie die scheinbar gesunden Kopftheile gleichfalls in den Krankheitsprozess hineingezogen sind, wenn auch freilich der Verlauf des Prozesses an diesen Theilen ein wesentlich anderer ist). Allein man sieht auch gar nicht selten auf dem erkirankten Mittelkopf, hauptsächlich auf dem vordersten Theile desselben längere Lanugo-Haare, die selbst in den späteren Stadien der Krankheit, wo die Patienten mit Sorgfalt die wenigen langen Haare des Mittelkopfes hüten und nur selten die Scheere arbeiten lassen, dennoch die Spur der Scheere zeigen.

Das Verhältniss der Lanugo-Haare zum Gesammtausfall ist verschieden zunächst nach dem mehr oder weniger vorgerückten Stadium der Krankheit und nach der grösseren oder geringeren Gleichmässigkeit, mit welcher die verschiedenen Theile des Mittelkoples von dem Krankheitsprozess ergriffen sind. Je früher nach dem Eintritt der Pubertät das erste Stadium der Krankbeit begomnen hat, desto gleichmässiger pflegt in der Regel der Prozess den ganzen Mittelkopf zu afficiren; hier ist die Anzahl der LanugoHaare schon früh ziemlich beträchtlich, weil die verschiedenen Regionen des Mittelkopfes sich an ihrer Production gleichmässig betheiligen. Wo die Krankheit 5 oder 6 Jahre nach der Pubertät oder gar noch später entstebt, da ist das Fortschreiten des Prozesses vom ersten Stadium zum zweiten an den verschiedenen Regionen des Mittelkopfes nicht ein gleich rasches: und in diesen Fällen ist Anfangs die Anzahl der Lanugo-Haare gering, steigt auch 
nur allmählich. Bei dem täglichen Haarausfall meines Kopfes kam 18611 Lanngo-Haar auf 12 andere, 18621 auf 8, 18631 auf 4 , 18641 auf 2, 18651 auf $0,8,18671$ auf 0.7 .

Wo die Glatze schon sehr bedeutende Fortschritte gemacht hat, ist das Verhältniss noch ungünstiger: In 2 Fällen, wo ich allerdings nur für einen Tag die Zählung vomahm, kamen auf 1 normales Haar etwa 3 Lanugo-Haare.

Fasst man demnach den Entwickelungsgang der Alopecia pityrodes zusammen, so ergeben sich folgende Züge:

Bei einem gesunden Individuum im Alter von 15 Jahren oder darüber tritt eine Anfangs unbedeutende, allmählich an Intensität zunehnende Pityriasis capitis ein, welche den ganzen Kopf ziemlich gleichmässig befällt. Nach Verlauf von in der Regel 5 bis 7 Jabren bemerkt der Patient einen beträchtiicberen täglichen Haarausfall, als er sonst wahrgenommen hatte; sind die Patienten weiblichen Geschlechts, so fällt in diesem Stadium bereits die grosse Anzahl kürzerer Haare und somit das Dünnerwerden des unteren Theiles der Zöpfe und das rebellische Hervortreten vieler Haare aus der Mitte derseiben auf. Bei Männern erscheint der Haarboden noch eben so dicht wie früher. Nach weiteren 2 bis 4 bis 6 Jahren erscheinen einzelne Stellen des Kopfes, besonders in der Nähe der Höhe des Scheitels und der Stirn, lichter; die Zahl der ausfallenden Haare nimmt erheblich zu: unter dem Ausfalle findet sich auch bei Männern eine sehr bedeutende Anzahl von Spitzenhaaren; ausserdem zeigen einzelne Haare bereits den LanugoCharakter, sehr viele sind in ibrem Dickendurchmesser erheblich herabgegangen.

In diesem Stadium des Krankheitsprozesses bemerkt man sehr häufig ein eigenthümliches Verhalten der Pityriasis.

Icb habe bereits früher erwähnt, dass die Pityriasis nach meiner Ansicht gleichzeitig mit der Alopecie auftritt.

Wenn die Autoren den Beginn der Haarkrankheit um viele Jabre später datiren, so rührt nach meiner Meinung der Irrtbum daber, dass man bisher das erste Stadium der Krankheit: die allmähliche Abnahme der typischen Länge des Haares (bei unverändertem Dickendurchmesser) üb ersah; es wurde dieses Uebersehen durch die gebräuchliche kurze Haartracht der Männer begünstigt. - Die Veränderung des typischen Längen- 
wachsthums ergreift das gesammte Haupthaar, sie schreitet aber nach einigen Jahren auf dem Mittelkopfe raseher fort, als in den anderen Regionen; sie geht in diesen anderen Regionen in fast allen Fällen nicht über das erste Stadium hinaus, vielmehr bleibt die typische Länge dieser Haare durchschnittlich 4 bis 6 Zoll und darüber (was man am deutlichsten bei Frauen sieht), bis die später eintretende Calvities als neues Moment das Haarwachsthum verändert.

Die Pityriasis ist in den ersten Jabren gleich dej Haarkrankheit über den ganzen Kopf gleichmässig verbreitet; sie hält bei ihrer Entwickelung in Bezug auf ihre Intensität gleichen Schritt mit der Alopecie, so dass man in der Regel aus der Intensität der Hautabschilferung einen Schluss auf die gleiche Intensität der abnorm grossen Schnelligkeit des Haarwechsels machen kann; besonders gegen das Ende des ersten Stadiums der Alopecie ist es schon bei kurzer Beobachtungszeit deutlich nachweisbar, dass auch ai den verschiedenen Regionen des Kopfes die Stärke der Pityriasis gleichen Schritt hält mit dem Grade der Haarkrankheit. Allein zu dem oben angegebenen Zeitpunkte der Entwickelung der Krankheit bemerkt man bei den meisten kranken, dass die Hauptabschilierung ihre frübere Intensität nur auf dem mittleren Theile des Mittelkopfes beibehält, während sie auf der Höhe des Scheitels und in der Nähe der Stirn erheblich abnimml; es sind diess dieselben Stellen, welche beim weiteren Fortschreiten der Krankheit am frühesten kahl werden. Diese auffallende Abweichung der sonstigen Gleichmässigkeit des Fortschreitens in der Alopecie zeigt sich 1 oder 2 Jahre später auch auf dem mittleren Theile des Mittelkopfes, sobald diese Regionen gleichfalls in den vorgeruickten Giad des zweiten Stadiums eintreten. Die Alopecie schreitet gleichmässig weiter, bis zuletzt die gesammten Haare der kranken Regionen zu Lanugo werden, mit je einer Lebensdauer von einigen Wochen; die Haar-Producirung erlischt nicht, schon obne Lupe siebt man die feinen Härchen dicht neben einander stehend, die ganze Glatze bedecken und die sehr zahlreichen Härchen, welche an einem Tuche, mit dem man etwas derb ïber die Haut fährt, haften bleiben, beweisen allein schon ohne jede weitere Beobachtung die Kurzlebigkeit derselben. Erst die im höheren Alter eintretende Calvities macht dieser Production ein Ende. 


\section{3}

Die Pityriasis hingegen nimmt bei dem erwähnten Grade des zweiten Stadiums gleichmässig $a b$, und tritt an jeder Region des Kopfes, an welcher die Haare zum grőssien Theile schon den Charakter der Lanugo zejgen, für die ganze fernere Zeit sehr schwach auf.

Es ist wesentlich für die Beurtheilung des beiden Krankheiten zu Grunde liegenden Prozesses, dass die Schweiss-Production an den erkrankten Regionen zu keiner Zeit einè wahrnehmbare Veränderung zeigt: in den Anfangsstadien keine Vermehrung, in den späteren Jahren keine Verringerung; wenn die Pityriasis längst bis auf eine kaum merkbare Abschilferung beschränkt ist, zeigt sich die bezügliche Hautpartie oft genug mit reichlichem Schweiss bedeckt, aber die Schweiss-Production ist mitunter ebenso reich, zu einer Zeit, da sich noch sebr zahlreiche Pityriasis-Schuppen varfinden.

Die Alopecia pityrodes ist ron jeher als eine Atrophie der Cutis aufgefasst worden; die Calvities gab für die Auffassung eine erwünschte und bequeme Analogie. Eine Atrophie der Cutis ist allerdings das Ende des ganzen Prozesses und es ist auch das charakteristische Moment der Atrophie vorhanden: die allmählich immer grösser werdende Kurzlebigkeit der einzelnen von dem kranken Organ erzeugten Produkte.

Allein eine klare Einsicht in den Gang eines Prozesses ist mit dem Begriff der Atrophie nicht gewomuen, am allerwenigsten bei einem so zusammengesetzten Organ wie die Cutis. Noch viel weniger gibt der Begriff einen Anhalt für die Beurtbeilung der auch nur nächsten ursächlichen Momente.

Die Autoren nehmen als nächstes Moment der Alopecie (um welche es sich wesentlich in ihren Erörterungen bandelt, obwobl, wie aus den näheren Mittheilungen folgt, in der Regel die in Rede stehende Art der Alopecie gemeint ist) entweder eine Atrophie der Nerven (cerebrospinalen oder vasomotorischen) oder eine Atrophie der Capillaren an. Mikroskopische Untersuchungen, welche diese Annahme beweisen könnten, werden allerdings nicht beigebracht, aber an ihrer Stelle Raisomements, welche eine genaue Kenntniss des Entwickelungsganges des zweiten Stadiums der Krankheit beweisen.

Ich habe zweimal Gelegenheit gehabt, Präparate von Personen, 
die an der in Rede stebenden Krankheit litten und durch intercurrente Krankheiten (beide durch Typhus abdominalis) hinweggerafft wurden, zu untersuchen; ich habe vielfach Kopfnäute von Personen untersucbt, welche an einer sehr verwandten Krankheit, der Pityriasis tabescentium, litten. Die Frage, ob eine Atrophie der Capillaren vorliege, kann doch nur so entschieden werden, dass man feststelit, ob an den kleinsten Gefässen und Capillaren organische Veränderungen und schliesslich Verödungen eintreten. Man kann sich doch nicht damit begnügen, dass man eine gesunde und eine kranke Kopfhaut injicirt, von beiden an 2 gleich entsprechenden Stellen Schnitte macht und nun sieht, ob der Schnitt der gesunden Haut in Folge zahlreicher vorhandener Gefässe ein bunteres Bild gibt als der Schnitt durch die kranke Haut. Bei einer solchen Beweismethode sind die Feblerquellen so zahlreich und so bedeutend, dass man besonnener Weise auf die Differenz der Ergebnisse kein Gewicht legen kann. An den Gefässen selbst habe ich keine krankhaften Veränderungen wahrnehmen können und sie sind meines Wissens bei der in Rede stehenden Krankheit auch sonst nicht constatirt worden. Vergleicht man Schnitte durch die erkrankte Hautpartie mit solchen durch die Haut der normalen Schläfen-oder Hinterhauptsgegend, so findet man, in Bezug auf die Gefässe der Haarbälge und der Haut, völlig dasselbe Bild.

Ich fand allerdings, dass der Dickendurchmesser der einzelnen Schichten der Cutis verändert ist; vergleicht man Schritl für Schritt Schnitte von der Mittellinie des Kopfes auf der Höhe des Scheitels, wo die Haarkrankheit am meisten vorgesehritten ist, bis zum Hinterkopf, wo sich der normale Haarwuchs wieder zeigt, so findet man die Länge des Haarbalges, also die Entfernung von dem Grunde des Haarbalges bis zur Oberfläche der Cutis, an der normalen Haarstelle in der Pegel etwas, zuweilen viel, grösser als an der kranken; zuweilen allerdings ist eine Differenz gar nicht vorhanden.

Allein in allen Fällen findet man eine Differenzdes Dickendurchmessers der beiden unteren Schichten der Gutis: der Dickendurchmesser der Epidermis-Schicht ist an beiden Stellen gleich, hingegen ist an der kranken Hautstelle die Schicht des eigentlichen Cutis-Gewebes zwischen der Epidermis und dem Unterhaut-Zellgewebe erheblich verdünnt. Die Intensităt 
dieser Verdünnung hält gleichen Schritt mit dem Stadium der Haarkrankheit: diejenigen Stellen, an welchen sich noch viele Haare von normalem Dickendurchmesser finden, zeigen auch eine geringere Verkürzung des Dickendurchmessers der in Rede stehenden Schicht als die Stellen, an welchen sich zum grössten Theile nur noch Lanugo findet.

In der Regel zeigt nun die Feitsehicht an den kranken und gesunden Stellen gleichfalls eine Differenz: ibr Dickendurchmesser ist an den kranken grösser als an den gesunden; die Zunahme der Möglichkeit ist zwar oft nicht beträchtlich, oft aber recht erheblich.

Zur Feststellung dieser Verhältnisse mussten in der Regel Schnitte von verschiedenen Stellen ein und desselben Kopfes dienen; hierbei ist die Möglichkeit nicht auszuschliessen, dass an diesen Stellen die beiden unteren Schichten der Cutis von Anbeginn an, also vor Ausbruch der Krankheit, eine verschiedene Mächtigkeit hatten. Ich kann diese Möglichkeit nicht verneinen: prüft man einzelne Stücke der Kopfhaut bei Menschen, deren Kopfhaar normal ist, so findet man auch bei solchen Köpfen, deren einzelne Haare einen nicht unerheblichen Unterschied ihres Dickendurchmessers zeigen, niemals einen erheblichen Unterschied der Mächtigkeit der mittleren Schicht der Cutis; es kommt wohl vor, dass bei einzelnen Haarbälgen das Fettgewebe höber hinanf steigt, als bei anderen, allein man sieht nie, dass alle Haarbälge mehrerer benachbarten Harkreise solche Differenz von anderen zusammenstehenden Haarbälgen zeigen. Das Fettgewebe zeigt allerdings recht oft an verschiedenen Regionen ein und desselben Kopfes einen Unterschied, allein derselbe besteht weniger in einer Verschiedenheit der Mächtigkeit der Schicht, vielmehr in einer Verscbiedenheit der Gewebs-Zusammensetzung: auf dem ganzen Mittelkopf ist nämlich in diesen Fällen das. Faselgerüst des Fetlgewebes reicher und die einzelnen Faserziuge breiter als an den Schläfen und dem Hinterhaupt, eine Beobachtung, deren Analogon (aber in erheblich gesteigertem Grade) wir bald bei erkrankter Kopfhaut wiederfinden werden.

Es ist also immerhin möglich, dass die an den haarkranken Hautstellen wahrnehmbare geringere Mächtigkeit der mittleren Schicht der Cutis eine ursprünglich gegebene war; in diesem falle möchte ich annebmen, dass dieser ursprüngliche Zustand der Cutis zu- 
gleich die wesentlichste Prädisposition zu der in Rede stehenden Haarkrankbeit enthălt; eine weitere Untersuchung wird diess erörtern und namentlich feststellen können, ob die erblich überkommene Disposition zur Alopecia pityrodes an eine solche ursprüngliche geringe Mächtigkeit der mittleren Schicht der Cutis gebunden ist.

Vergleicht man genau entsprechende Knpíbautregionen an den beiden Seiten des Kopfes eines Individuums, das bei Lebzeiten an Alopecia pityrodes gelitten hatie, so findet man einzelse entsprechende Stellen, an welchen die Harkrankheil einen wesentlich verschiedenen Grad des zweiten Stadiums zeigt: auf der einen Seite noch viel dickes Haar, auf der anderen sebon meist Wollhaar, man findet an solchen Partien gleichfalls die erwähnte Verdünnung der mitlieren Schicht der Cutis in einem dein jedesmaligen Stadium der Harkrankheit entsprechenden Grade. Es ist natürlich nicht gestattet, aus dieser entsprechenden Gleichmässigkeit einen Grund gegen die Annahme einer ursprünglich gegebenen Dünnheit der Mittelschicht der Cutis herleiten zu wollen: die ursprüuglich gegebene Disposition kann auch an den beiderseitigen entsprechenden Kopfhautstellen sehr gut eine verschiedene sein.

Die haarkranken Kopfhautstellen zeigen in der Fettschicht der Cutis ausser der erwähnten Abweichung in der. Mächtigkeit derselben noch eine Abweichung in ihrem Bau und zwar dieselbe Abweichung, welche nach der, obigen Mittheilung sich auch bei haargesunden Personen auf dem Mittelkopfe findet. Es wird uämlich das Fasergerüst des Fettgewebes reicher und die einzelnen Züge des Gerüstes werden breiter, jemehr man sich von einer haargesunden Stelle denjenigen Regionen nähert, welche hauptsächlich nur noch Lanugo zeigen. Diese Veränderung ist constant; man vermisst sie an keiner haarkranken Stelle.

Nach meinen an Lebenden gemachten Beobachtungen möchte ich schliessen, dass diese letzterwähnte Veränderung in dem Fetigewebe der Cutis sehr früh beginnt, gleich mit dem Anfaing des ersten Stadiums der Alopecie, und dass sie langsam wie diese vorschreitet. Prüft man viele haargesunde Köple, so findet man, dass bei den meisten die Haut auf dem Mittelkopf sich leicht in einer Falte aufheben lässt, das Fettgewebe biluet also hier ein 
lockeres Mittelglied $z$ wischen der eigentlichen Cutis und den tieferen:Geweben, wenn auch die Verbindung am Mittelkopf nur selten so locker ist wie an den schlälen oder dem Hinterhaupt. Bei der Alopecia pityrodes hingegen zeigt sich die Haut auf dem Mittelkopf schon sehr früh fester angeheftet; schon zu einer Zeit, wo das Verhältniss der Spitzenhare zum Gesammausfall wie $1 \mathrm{zu}$ 10 ist. Es ist diess Moment, das, wie erwähut, nach meinen Erfahrungen nie feblt, als ein diagnostisches fiur die Krankheit iberhaupt sehr bequem, wenn es auch keinen Anhalt für die grössere oder geringere Intensität des Leidens gibt; der Grad der Intensität ist nur durch Zählung des Haarausfalls und durch Feststellung des Verhälthisses der Spitzenhaare zum Gesammtausfall mit Sicherheit zu diaguosticiren.

Wenn es hingegen zunäcbst nur darauf ankommt, das. Vorhandensein der Krankheit zu erkennen, so kann man dieselbe annehmen; sobald die strafiere Anheftung der Kopfhaut sich neben einer auch nur mässigen Schuppung der Koplhaut zeigt.

Es wäre für die Erkenntniss des Entwickeluugsganges der Krankbeit und auch für die Therapie von grosser Wichtigkeit, wenn man deutliche Differenzen im Verhalten der Cutis zwischen einer Kopthaut, welche das erste Stadium der Alopecie zeigi, und einer solchen des zweiten Stadiums nachweisen könnte, da es für Mällner besonders, hauptsächlich darauf ankommi, die Abnahme des Dicliendurchmessers der einzelnen Haare, also das Eintreten des zweiten Stadiums, zu verhindern. Wie ich in dem ersten Aulsatz erwähnt habe, zeigen alle diejenigen Personen, die an Alopecia pityrodes leiden, auch an den Schläfen und am Hinterbaupte das erste Stadium der Krankheit. Ich habe an diesen Hautstellen eine Abweichung von der entsprechenden haargesunder Köple nicht finden können; zum Vergleiche wählte ich haargesunde Kopfhäute von gleicher Dicke: ich fand jedoch keine Verschiedenheit in der Dicke der beiden unteren Sebichten. Es darf indess aus diesem tegativen Ergebniss keineswegs der Schluss gemacht werden, dass auch auf dem Mittelkopl während des ersten Stadiums der Alopecie Veränderungen der Cutis fehlen, weił die Krankheitszustände auf dem Mittelkopfe und den Schläfen zwar sebr ähnlich jedoch nicht identisch sind:- die Schläfen- und Hinterbauptsgegend zeigen die Pityriasis und das abriorine Verliältniss der ausfallenden Archiv f. pathol. Anat. Bd. XLI. Hft. 3 u, 4 . 
Spitzenbare (gleich dem Mittelkopf), allein nie kommt es an jenen Gegenden zu einer Verringerung des Dickendurchmessers der Haare und nie zu einer straffen Anheftung der Cutis; es darf daher wohl vorausgesetzt, werden (da diese straffere Anheftung der Cutis, wie ohen erwähnt, auf dem Mittelkopf schon im Anfang des ersten Siadiums wahrnehmbar ist), dass nicht völlig derselbe Prozess an den verschiedenen Regionen des Kopfes stattfindet.

Die angeführte Abnahme der Mächtigkeit der mittleren Schicht der Cutis betrifft nach meinen Messungen wesentlich denjenigen Theil derselben, welcher unterhalb der Einmündung der Talgdrüsen in den Harbalg liegt. In den vorgerückten Stadien der Krankheit ist diess so deutlich, dass es keiner Messung bedarf: man sieht hier auf den ersten Blick, wie abnorm nahe die Talgdrüsen an die Fettschicht der Cutis gerückt erscheinen. Der Abstand zwischen Talgdrüsen und Epidermis zeigte sich bei zablreichen Vergleichen und Messungen an kranken und gesunden Stellen unverändert.

Die Frage, in welchem Grade die Talgdrüsen if den Krankheits-Prozess hineingezogen sind, lässt sich an Lebenden durch die Untersuchung der Pityriasis-Schuppen beantworten. Wir müssen zu diesem Zwecke auf die Frage über die Ursprungsstätte der Pityriasis-Schuppen kurz eingehen. In einer deutlichen Uebersicht hat Hebra in den Eläuterungen zu seinem Atlas der Haarkrankheiten die bezügiichen Ansichten der Autoren aufgezählt. Die meisten sehen in den Pityriasis-Schuppen Epidermis-Reste. Hebra kommt nach seinen sehr zablreichen Beobachtungen zu dem Schluss, dass die Schuppen wesentlich aicht Abschilferungen der Epidermis sondern hauptsächlich abnormes Secretions-Product der Talgdrüsen sind; er stützt diese Ansicht durch die Thatsache, dass man bei zahlreichen Beobachtungs-Objecten schrittweise alle Uebergangsstufen von geringer Pityriasis capitis bis zur Seborrhoe fände und dass man auch bei ein und demselben Individuum zuweilen ein solches allmähliches Anwachsen der einlachen Pityriasis zur Seborrhoe wahrnehmen kann. Er lässt daher die Bezeichnung Pityriasis fallen, ersetzt sie durch Seborrhoe und nennt die (gewöhnliche) Pityriasis capitis nach dem Vorgang der Franzosen Seborrhoea sicea. (Flux sébacé sec).

Die beiden von Hebra angeführten Thatsachen sind richtig, seiner Nomenclatur indess kann ich nicht beistimmen. Ich habe 
die Schuppen von meinem (an Pityriasis leidenden) Kopfe mit einem nassen Staubkamm. vorsichtig entfernt, getrocknet, gewogen, in Aether gekocht, dann den Rückstand gewogen und mikroskopisch untersucht. Das Resultat von sieben solchen Wägungen war, dass der Gewichtsverlust der Schuppen durch die Bebandlung mit Aether im Durchschnitt drei Fünftheile der früheren Masse betrug; zwei Fünftheile waren Epidermis. Ich habe dieselben Wägungen an Pityriasis-Schuppen vorgenommen, die ich von den Haaren anderer Personen abkämmte, ohne mit dem Kamm die Kopfhaut selbst zu berühren (es betraf Personen, die an Alopecia pityrodes oder Pityriasis tabescentium litten): das Resultat war gleich oder sehr ähnlich dem an den Schuppen meines Kopfes gefundenen. Ich hatte zweimal Gelegenbeit, eine Complicirung der Pityriasis capitis mit Seborrhoe zu beobachten: die Seborrhoe trat acut und intensiv auf und veriief so, dass in dem einen Fall nach vierzehn Tagen, in dem anderen nach drei und einer halben Woche die alte Pityriasis übrig blieb. Ich habe die Borken und Schuppen in dem ersten Fall jeden dritten Tag, im zweiten jeden zweiten Tag untersucht: auf der Höhe der Krankheit betrug der nach der Behandlung mit Aether zurückbleibende Rest der Epidermis-Partikeln den neunten, resp. elften Theil der untersuchten Borke, nach Ablauf des Prozesses fand sich wieder das alte Verhältniss von 2 zu 5. Wo der Seborrhoe-Belag fast flussig ist und die Haut wie mit 0 el bestrichen erscheint, ist der Gewichtsantheil der Epidermis gewiss noch kleiner, für die Entscheidung der Ursprungsstätte der gewöbnlichen Pityriasis-Schuppen ist jedoch auch dieses Gewichtsverhältniss obne Einfluss. Es bestehen sonacb die Schuppen der Pityriasis eapitis zur grösseren Hälfte ihres Gewichtes aus krankhaft veränderten (abnorm festen) Secretions-Producten der Talgdrüsen, bei der Bezeichnung der Krankheit ist man daher (so lange man eine symptomatische Benennung wählen muss) zu einer Wahl genöthigt, falls man nicht einen Doppel-Ausdruck brauchen will; ich möchte vorschlagen, dass man den Ausdruck Seborrhoe nach wie vor auf die gewöhnlich mit diesem Namen bezeichneten Zustände beschränkt und die in Rede stehende Krankheit auch fernerbin Pityriasis nennt; man muss sich dann bei dieser Bezeichnung allerdings bewusst bleiben, dass es sich nicht allein um eine gesteigerte Abschilferung der Epidermis handelt. 
In dieser Betrachtung über das Verhäliniss der Pityriasis zur Seborhoe und in den oben angeführten Beobachtungen über die Gleichzeitigkeit der Lutstehung der Pityriasis und der Alopecie liegt für mich der Grund für die Bezeichnung der in Rede stehenden Haarkrankheit als Alopecia pityrodes. E; liegt in dieser Bezeichnung zugleich ein deutlicher Hinweis, dass beide KrankheitsErscheinungen nur Sympiome resp. Resultate ein and desselben Krankheitsprozesses sind.

Differential-Diagnose der Alopecia pityrodes von den verwandten Krankheitsauständen.

Maii unterscheidet seit alter Zeit vier Arten von Krankheiten der Kopliaare:

Die Atrichie, den angeborenen Mangel (gänzlichen oder theilweisen) der Haare;

Die Calvities, das Ausfallen der Haare ii Folge vorgerücktei Alters;

Das Defluvium capiliorum und

die Alopecie.

Die Abgrenzung zwischen Defluvium capillorum und Alopecie ist bei den verschiedenen Autoren eine verschiedene und bei den meisten nicht scharf. Im Allgemeinen versteben die Autoren unter Defluvium capillorum ein am ganzen Kopfe plötzlich und ziemlich śleichmässig aufretendes Ausfallen der Haare, ohne wahrnehmbare Erkrankung der Kopfbaut, in der Regel nach vorausgegangener acuter oder chronischer Erkrankung des Gesammt-Organismus. Mit Alopecie bezeichnen sie ein Ausfallen der Haare in Folge örtlicher Erkrankung.

Die meisten Autoren unterscheiden vier Arten der Alopecie je nach der verschiedenen Art der örtlichen Erkrankung der Cutis: Alopecił durch Entzündung der Haut oder der Bälge (Erysipel, Acre);

Durch Parasiten, welche die Haare oder selbst die Haarbälge zerstören (Herpes tonsurans, Tinea);

durch den Druck von Geschwülsten;

durch örtliche Nerven-Erkıankung (Area Celsi).

Das Ausiallen der Haare bei allgemeinen chronischen Erkrankungen wird bald zum Defluvium capillorum bald zur Alopecie 
gezählt, es gilt diess namentlich von den bei Syphilis und bei tabescirenden Kranken vorkommenden Erkrankungen des Kopfhaares.

Bei unserer noch so sehr geringen Einsicht in die den verschiedenen Anomalien des Haarwachsthums zu Grunde liegevden pathologischen Zustände der Cutis scheint es mir nicht angemessen, die usuell gewordenen Bezeichnungen für die Haar-Erkrankungen umzumodeln; wir mussen die symptomatischen Bezeichnungen noch festhalten, weil sie wenigstens einigermaasen orientiren. Dieser letzte Grund mag es rechtfertigen, dass ich in der Folge einige weitere Unterarten der Alopecie aufstelle.

Die Krankheitszustände, welche zu einer Verwechslung mit Alopccia pityrodes Veranlassung geben, sind:

die Calvities praematura,

die Alopecia eczematodes, nachdem das Eczem längere Zeit aufgehört hat zu nässen,

die Alopecia rheumatica und

einzelne Formen der bei Syphilis vorkommenden Erkrankung der Haare.

Ich habe in dem früheren Aufsatz*) ein Beispiel von der Veränderung des typischen Haarwechsels angefubrt, welche bei der Calvities eintritt. Der Haarausfall bej diesem, im Jahre 1860 52 Jahre alten, Herrn betrug täglich im Minimum 25, im Maximum 34, im Mittel 29; 1861, im Minimum 22, im Maximum 34, im Mittel 28; 1862, im Minimum 23, im Maximum 36, im Mittel 26; im Jahre 1863, also im 55. Lebensjabre machten sich zuerst die Erscheinungen der Calvities deutlich bemerkbar. Der tägliche Haarausfall betrug damals im Minimum 45, im Maximum 77, im Mittel 58; die Spitzenhaare verhielten sich zum Gesammtausfall wie 1 zu 3,103. Dabei fanden sich bereits eine nicht unbedeutende Anzahl von Spitzenhaaren mit sehr geringem Dickendurchmesser, im Durchschnitt war jedes zwölfte ausfallende Haar ein LanugoHaar. - Das folgende Jahr zeigt einen täglichen Haarausfall von im Minimum 82, im Maximum 116, im Mittel 101. Die Spitzenhaare verbielten sich zum Gesammtausfall wie 1 zu 2,324; es war. etwa jedes achte ausfallende Haar ein Lanugo-Haar. - 1865

*) Bd. XXXVII dieses archivs S. 23. 
betrug das Minimum des täglichen Ausfalls 109, das Maximum 163, das Mittel 137; die Spitzenhaare verhielten sich zum Gesammlausfall wie 1 zu 2,068; es war im Durchschnitt jedes sechste ausfallende Haar ein Lanugo-Haar.

Es erfolgt sonach beim Beginn der Calvities eine rasche Steigerung des absoluten Haarausalls, eine ebenso rasche Zunabme des Ausfalls der Spitzenbare und eine schnelle Abnatime des Dickendurchmessers vieler Hare. Die Erscheinungen, welche in der Alopecia pityrodes durch weite Zeiträume auseinander gehalten sind, rücken bei der Calvities nahe zusaminen; besonders avffallend war mir die Thatsache, dass innerhalb eines Jahres eine so beträchtliche Anzahl von. Haaren an ihrer typischen Dicke so erhebliche Einbusse erlitten. Ich habe in zwei andercn Fallen eine gleich rasche Veränderung selbst inmerhalb eines Zeitraums von 7 und 8 Monaten gesehen. Es müssen erhebliche Veränderungen der Gutis sein, welche eine so rasche Veränderung der typischen WachstbumsVerbältnisse bedingen. Ich habt ein so schnelles Vorschreiten der Ernährungs-Störung selbst in den Fällen von Alopecia pityrodes nicht gesehen, in welchen die Krankheit unmittelbar nach Eintritt der Pubertät begann und wo schon nach wenigen Jahren eine bedeutende Glatze sich ausbildete, 2 s steigt allerdings in diesen Fällen der lägliche Haarausfall sehr beträchtlich, allein es vergehen doch 2 bis 3 Jahre, ehe sich Lanugo-Haar im täglichen Ausfall zeigt.

Eine genaue Untersuchung am Kopfe des Lebenden zeigt, dass beim Boginn der Calvities die Bildung der Lanugo-Hare, $z$ war auf dem ganzen Kopf erfolgt, allein nicht in gleicher Weise auf den verschiedenen Regionen des Kopfes. Man bemerkt nämlich (am bester bei kurzgeschorewen Köplen) am ganzen Kopf, mit Ausnabme einer Stelle die Lanugo-Haare ziemlich spärlich, so dass ein Lanugo-Haar erst etwa in jedem fiunten oder sechsten Haarkreise sich findet. An einer Stelle hingegen stehen die LanugoHaare dichter und nach einigen Monaten so dicht, dass an dieser Stelle in jedem Haarkreise bereits zwei Wollhärchco vorhanden sind. Es befindet sich diese Stelle fast immer auf der Höhe des Scheitels und breitet sich allmählich nach vorn, später nach den Seiten und nach hinten aus. In den Fällen, die ich mehrere Jahre hindurch beobachten konnte, stand die langsame Ausbreitung der 
Erkrankung in einem auffallenden Gegensatz zu dem so rasch erfolgten Abfall des Dickendurchmessers der Haare an jener Stelle; allein die Erkrankung bewahrte auch an den neu ergriffenen Regionen denselben Charakter, es hatte verhältnissmässig lange gedauert, ehe an der neuen, der kranken dichtbenachbarten Stelle mehrere-Wollhärchen vorkamen, allein war diess einmal geschehen, so ergriff der Prozess die neue Region sehr intensiv und sie wurde in einigen Monaten kahl. Die Region, welche a uf einmal ergriffen wurde, hatte im Durchschnitt etwa die Grösse von einem Zweisilbergroschenstïck.

Bei denjenigen Männern und Frauen, deren eirzelne Haare eine fast gleichmässige Dicke ihres Querdurchmessers haben, erfolgt an der ergriffenen Stelle die Ausbildung der Glatze früher als bei den Leuten mit Haaren von ungleichmässiger Dicke - ein Gegensatz gegen die bei Alepecia pityrodes gemachte Beobachtung. Es scheint, als wäre die ungleiche Kräftigkeit der Einsassen ein und desselben Haares ein Schutz gegen das rasche gl ejchmässige Erliegen unter der Macht des Krankheitsprozesses. Man muss auf diese Ungleichmässigkeit Rücksicht nehmen, um zu begreifen, warum in dem einen Fall von Calvities (hierher gehört der oben angeführte) eine verhältnissmässig längere Frist vergeht, bevor (trotz erheblicher Zunahme des Gesammtausfalls der Spitzenund Lanugo-Haare) der Anfang einer Glatze sich erst verhältnissmässig spät zeigl, hingegen in dem anderen Falle verhältnissmässig früh.

Nie sah ich bei der Calvities eine auch rur mässige Pityriasis, abgesehen von den Fällen, in welchen es sich um tabescirende Individuen handelte, welche dann allerdings gleich jungeren Personen die Pityriasis tabescentium zeigten. Geringe Abschilferungen der Epidermis der Kopthaut kamen allerdings zuweilen vor.

Auch die bei der Alopecia pityrodes beschriebene straffe Anbeftung der Kopfhaul fehlt bei der Calvities; im hohen Alter allerdings tritt, wie die mikroskopische Untersuchung lehrt, eine erhebliche Veränderung der Kopfhaut ein, welche zwar an die bei der Alopecia pityrodes beschriebene erinnert, aber doch mit ihr nicht identisch ist, es erscheint zwar die mittlere Schicht der Cutis verdünt (als Massstab diente die Dicke der Epidermisschicht, welche am wenigsten variirend erscheinb), allein auch das Fettgewebe 
hatte an Mächtigkeit eingebüsst und eine Massenzunahme des Fasergerüstes dieses Gewebes war nicht bemerkhar. Dazu kommt, dass die Calvities erst in höheren Manuesalter eintritt, eine Verwechslung derselben mit Alopecia pityrodes wird selten erfolgen könmen.

Allein es gibt eine nicht geringe Anzabl von Individuen, bei welchen ein allmähliches Kablwerden des Kopfes schon früh unter Erscheinungen eintritt, welche den bei Calvities bemerkbaren gleichen; man hat diesen Zustand sehr passend Calvities praematura genannt.

Es unterscheidet sich der tä̧liche Haarausfal! bei der Calvities praematura von dem bei Calvities senilis dadurch, dass bei ersterer die Zunahme des absoluten äglichen Harausfulls gering ist und dass an den Schläten und dem Hinterhaupt Lanugo nicht erzeugt wird; es kommt zur Lanugo-Bildung nur an einel einzigen kleinen Stelle (in den von mir beobachteten Fällen immer die Höhe des Scheitels). Gemeinsam aber ist beiden Arten der Calvities die eigenthünliche Art des Fortschreitens des Prozesses: die Krankheit ergreift die benachbarten Haarkreise erst, sobald die unmittelbar vorber ergriffene Region schon zum grössten Theil nur noch Lanugo zeigt.

Von der Alopecia pityrodes unterscheidet sich die Calvities praematura durch die bei der Alopecie vorhandene beträchtliche Zunahme der ausfallenden Spitzenhaare, durch die bei der Calvities fehlende Pityriasis capilis und die gleichfalls fehlende straffere Anheftung der cutis. Ganz besonders unterscheidend ist der Verla uf beider Krankheitszustände. Bei Calvities praematura zeigt die Höhe des Scheitels schnn eine kleine Glatze; während die übrigen Haarkreise des Mittelkopfes noch keine Abnabme des Dickendurchmessers ihrer Einsassen wahrnehmen lassen; - bei der Alopecie erfolgt die Verdünnung des Haarbondens und die Bildung der Glatze auf dem ganzen Mittelkopf, bis nahe zur' Stim ziemlicb gleichmässig, an allen einzelnen Regionen des Mittelkopfes findeu sich fast gleichmässig dieselben krankhaften Veränderungen.

Wo sich zur Calvities praematura eine Pityriasis tabescentium binzugesellt (ich habe einen solchen Fall nur einmal genaner beobachtet), da ist eine differentielle Diagnose zwischen Calvities praematura und Pityriasis pityrodes nicht mehr möglich, weil, wie 
ich oben wiederholt bemerkt habe, mit jeder Pityriasis eine Abnahme des typischen Längenwachsthums der Haare verbunden ist. In dem einen von mir beobachteten Falle schritt die Calvities rasch fort and es traten auf dem ganzen Mittelkopf fast in jedem Haarkreise Lanugo-Haare auf. Jn einem solchen Fall ist das Auseinanderbalten der Diagrose prognostisch und therapeutisch natürlich ohne Bedeutung.

In der Regel wird die differentielle Diagnose beider Krankheitszustände leicht sein: es ist bei der Verschiedenheit der zu Grunde liegenden Prozesse auf diese Unterscheidung Gewicht zu legen, weil die Gelegenbeit zu anatomischer Untersuchung in den ersten Stadien der Krankheiten sich selten findet und einè genaue Beobachtung des Verlaufs wohl noch vielfach Aufklärung wird geben können. Therapeutische Versuche habe ich bei Calvities praematura nicbt gemacht, schon die ausserordentlich rasche Abnahme der Kräftigkeit der Haare ein und desselben Kreises lässt die Therapie aussichtslos erscheinen.

Die $\mathrm{z}$ weite Krankheit, welche zur Verwechslung mit Alopecia pityrodes Veranlassung gibt, ist die Alopecia eczematodes in ihren späteren Stadien.

Es ist ein Verdienst Hebra's, zuerst allgemein bekannt gemacht zu haben, dass das Eczem in seinen späteren Stadien in sehr vielen Fällen nicht mehr den Charakter eines Bläschen-Ausschlages, sondern den eines Schuppen-Exanthems hat. In einzelnen Fällen ist der Verlauf dieses späteren Stadiums eigentbümlich, es tritt nämlich ein fast vollständiger Rückgang des Leidens ein, dann nach einer Pause von vielen Monaten gewinnt die schuppenartige Flechte wieder an Intensität und behält diese Intensität fast unverändert ein Jahr oder mehrere hindurch, um (wenigstens in den Fällen, die ich gesehen habe), in vollständige Genesung übergehen.

Dieses Stadium der Krankheit ist es, welches eine Verkennung des Grundprozesses leicht möglich macht. Es ist bekanntich das Eczema capitis, sobald es einige Wochen oder Monate gedauert bat, mit einem Defluvium capillorum verbunden; die Haare fallen an den erkrankten Stellen massenhaft aus, in verhältnissmässig. knarzer Zeit erscheint ein grosser Theil des Kopfes haarlos. In der Regel stellt sich der Nachwuchs des Haares nicht früher ein, als bis das Bläschen-Stadium des Eczems, vollständig vorüber ist. 
Dann spriessen jedoch die Haare sehr reichlich, zeigen fast immer den früheren Dickendurchmesser und die frühere Farbe. Allein es gibt Fälle, in welchen der Nachwuchs der Haare so früh nich eintritt; es sind diess (wenigstens nach meinen Beobachtungen) nur solche Fälle, in welchen das spätere flechtenartige Stadium des Eczems eine lange Dauer zeigt. Hier ist die Art des Haarnachwuchses eine andere, es entsteht kein Haar von normaler typischer Länge, sondern der Nachwuchs ist kurz und kurzleigig und tritt auch nur in einzelnen zerstreuten Posten auf. Ihre typische Dicke hingegen haben die einzelnen Hare an den meisten Stellen behalten; Lanugo findet sich nur ausnahmsweise. Der erste Nachwuchs fält nach einigen Monaten aus, der spätere ist noch dürftiger. Er bleibt mangelhaft Monate oder Jahre hindurch, dann tritt unter völligem Verschwinden der Reste des Eczem's allmählich normale Haarbildung ein.

Der Verlauf solcher exceptionellen Fälle von Eezema capitis lässt sich genau nur an Kranken verfolgen, die man Jahre lang fast beständig unter Augen hat. Die Fälle scheinen nicht sehr bäufig zu sein; ich selbst habe nur zwei behandelt, die ich hier kurz erwähnen will.

Ein Diensträadchen bekommt 1858 Eczema capitis; die Bläschenbildung zieht sich etwa ein Jahr lang hin, wäbrend der Zeit was das vorher reiche Haar ausgefallen, der Kopf erschien Kahl, nur hinter den Ohren, in der Mitte des Hinterhaupts und nahe an der Stirn standen einzelne Haarbüschel; einzelne Haare zerstreut an den übrigen Kopftheilen. Gegen Ende 1859 hört die Bläschenbildung auf, der Bopf bedeckt sich ziemlich gleichmässig mit kurzen Haar, zwischen welchem sebr reichliche Schuppenbildung auftritt. Die Schoppenbildung nimmt Anfang 1860 zu, das neu gebildete Haar fälit aus; Mitte 1860 ist der Kopf wieder fast ganz kahl, es zeigt sich auch kein neuer Haarwuchs. Die Pityriasis zeigt wiederbolte Abnahme and Zunahme, ohne dass Haare von normaler Dicke entstehen; eine genaue Besichtigung zeigt an der ganzen Kopfhaut gleichmässig sehr zahlreiche Wollhärchen, die etwa eine Linie über die Kopflaut bervorragen. An der Grenze des früberen Haarwuchses, besonders in der Gegend um beide Ohren, entsteht $a b$ und zu ein Schub von Eczembläschen. Dieser Zustand bleibt unverändert bis Mitte 1863; im Sommer dieses Jahres tritt am ganzen Kopfe gleichmässig die Bildung von kräftigem Haar ein, die Schuppung der Kopfhaut verschwindety nur das rechte 0 hr erscheint noch geröthet und mit Schuppen bedeckt. Die Haarbildung schritt gleichmässig fort; im Januar 1867 zeigte der Kopf gleichmässig ziemlich reiches, sechs- bis achtzehn Zöll ]anges, Haar.

Das Mädchen war beiro Ausbruch der Krankbeit 24 Jatre alt. 
Es waren nach einander angewendet worden: methodische Einreihung der Schmierseife, Veratrin in Salbenform und spirituöser Lösung, Tannin, Zincum sulphuricum, Benzeë-Tinctur, zum Schluss zehn Wochen lang Cuprum sulphuricum in Lösung und Cuprum oxydatum nigrum in Salbenform.

2. Ein an Hämorrhoidalbeschwerden leidender Raufmann beliommt in seinem 32. Lebensjabr (1860) ein Eczema capitis et faciei; schon nach 4 Monaten ist der Mittelkopf und ein Theil der Schläfen- und der Hinterbauptsgegend kahl. Das Eczem verliert schon nach 6 Monaten seinen Bläschencharakter und wird schuppig. Die Haare kcommen nun allmäblich hervor, erheblick weniger dicht als früher, jedoch das einzelne Haar von der gewöhnlichen Dicke, sie fallen nach 3 bis 4 Monaten wieder aus. Der Nachersatz ist zum kleineren Theil von normaler Dicke, zum grössern Theil Lanugo, die typische Länge ist sehr gering (ein viertel Zoll bis einen Zoll). Die Cutis bleibt hyperämisch, mitunter treten an einzelnen Regionen des Kopfes. und Gesichtes anstatt der Schuppen neue Eczembläschen auf. Dieser Zustand dauert bis zum Ende des Jahres 1804. Dann erfolgt, zunächst in der Năhe der Stirn, bald am ganzen Kopf gleichmässig und ziemlich dicht die Bildung von normal dickem Haar. Im Mai 1867 erscheint der Haarwuchs normal. Das Eczem ist verschwunden bis auf eine zurückgebliebene kleienartige, ziemlich beträchtliche Abschuppung beider äusseren Gehörgänge.

Therapeutisch waren angewendet worden die Bäder von Marienbad, Ems, Kissingen, zuletzt ein Seebad. Oertlich: Schmierseife, Cuprum sulphuricum, Cupram oxydatum nigrum, Benzoëtinktur, zuletzt Tannin.

Erhebliche Disposition zu Haut- oder Haarkrankheiten war nicht vorhanden; an Syphilis hatte. Patient nie gelitten; Hämorrboidalbeschwerden sind in der Familie, welcher Patient angehört, einheimisch.

Diese beiden Fälle sind für die Wachsthums-Verhältnisse des Kopfhares nach meinem Erachten besonders darum sehr lehrreich, weil sie beweisen, dass unter Umständen ein Haar, welches an seinem Dickendurchmesser sehrerhebliche Einbusse erlitten hat, wieder zur Norm zurückkehren kann. Nach meinen, an Syphilitischen gemachten Beobachtungen, bin ich zu der Annahme geneigt, dass eine solche Wiederkehr des normalen Dickendurchmessers nur in den Fällen vorkommt, in welchen das Haar an seiner typisehen Länge sehr rasch die erheblichste Einbusse erleidet; die Veränderungen der Cutis, welche in einem solchen Fall die krankhafte Haarbildung bedingen, müssen zwar tief eingreifend sein, allein die Plötzlichkeit ihres Auftretens scheint die Möglichkeit einer völligen Pestitution in integrum zu involviren.

Dass die völlige Wiederherstellung des normalen Zustandes selbst nach einer Krankheitsdauer von vier Jahren eintrat, macht 
mir die in der Literatur verzeichneten Fälle von Genesung nach selbst viel längerer Krankbeit glaublich; ich werde auf einige dieser Fälle bei der Therapie der Alopecia pityrodes noch kurz zurückkommen. Die Diagnose zwischen Alopecia pityrodes und solchen Fällen von Alopecia eczematodes ist gewiss nicht immer leicht zu stellen, zumal wenn man nicht die Entwickelung der Krankheit gesehen hat. Diejenigen Krankheitsfälle, welche mir vorgekommen sind, machten keine diagnostischen Schwierigkeiten. Die Ausbreitung der Krankheit über einen so grossen Theil des Kopfes (während die Alopecia pityrodes nur den Mittelkopf ergreift), die grössere Intensität der Abschuppung, das Vorhandensein von Eczem Bläschen an einzelnen Tbeilen des Kopfes oder des GesichŁes, die Gleichmässigkeit des Befallenseins der einzelnen Haarkreise in allen ihren Einsassen ermöglichten die Diagnose der Alopecia eczematodes scbon bei der ersten Untersuchung. Ich glaube auch, dass man in den meisten Fällen, die hierher gehören, nicht lange im Zweifel bleiben wird.

Verwechselungen beider Krankheiten können hingegen leichter eintreten, sobald sich zur Alopecia pityrodes eine Seborrhoe hinzugesellt, was nicht selten geschieht.

Ich habe bereits oben erwähnt, dass die Bezeichnung Alopecia eczematodes nur als Nothbebelf gewählt ist; ich weiss jedoch zur Zeit keine bessere; eine Ausscheidung dieser Krankheitsart ist nothwendig und die gewählte Bezeichnung orientirt doch wenigstens über eine in die Augen fallende Complication der Krankheit, wenn auch nicht über eine Ursache derselben.

Dasselbe gilt von der nun folgenden Art der Alopecie, der Alopecia rheumatica. Ieh habe vor dieser Krankheit uberhaupt nur drei Falle gesehen und von diesen nur einen Jahre lang beobachtet.

Fin Mann von (im Jahre 1858) 35 Jahren batte in den vorausgegangenen Jahren vielfach an ragem Mnskel-Rheumatismus gelitten; im Mărz 1858 erkrankte er an einem fieberhaften Rheumatismus der Nackenmuskeln; noch während der Reconvalescenz traten heftige Schmerzen auf dem ganzen Mittelkopf auf, welche durch Berübrung gesteigert wurden. Die Haut auf dem Mittelkopfe war weder geröthet noch geschwolien, doch hatte Patient an den leidenden Steilen das Gefühl beträchtlicher Hitze; der nachfühlenden Hand orschien die Temperatur der kranken Region nicht erhöht. Die Schmerzhaftigkeit danerte im ganzen zwei Jahre; Wochen lang war sie sehr mässig, dann folgten wieder Exacerbationen, allein auch während 
der Zeit der Remission wurde jeder mässige Druck schmerzhaft empfunden. Die Empfindlichkeit war an der ganzen erkrankten Stelle eine gleichmässige.

Dem sechswöchentlichen Gebrauch zu Wiesbaden folgte Genesung von den Schmerzen; doch bestand noch längere Zeit grosse Empfindlichkeit gegen kühle Luft.

Die Diagnose der Krankheit als einer rheumatischen ist keine absolut sichere; der chronische Rheumatismus der Kopfschwarte ist ja überhaupt für viele Aerzte eine Krankheit von zweifelhafter Existenz und die Differential-Diagnose von Neuralgien schwierig. Indess die gleichmässige Verbreitung des Scbmerzes über den ganzen Mittelkopf, der Mangel an Druckpunkten, das Fehlen jeder Irradiation, der Mangel an vollständigen Intermissionen veranlassten mich zur Annahme eines rheumatischen Leidens.

Das Haar verhielt sich bei dieser Erkrankung folgendermaassen: etwa sechs Wochen nach dem Beginn der Krankheit trat ein wabres Defluvium capillorum ein, nur dass auch hier wieder nicht alle Einsassen des einzelnen Haarkreises ergriffen wurden; es blieben je ein oder zwei Haare stehen und gingen auch bei einem auf sie einwirkenden mässig kräftigen Zuge nicht aus. Der nachfolgende Nachwuchs war spärlich, kurzlebig und von verkleinertem Dickendurchmesser, doch trat Lanugobildung nur vereinzelt auf. Im Laufe des nächsten Jahres erfolgte die Lanugobildung reichlicher und es waren nun fast in jedem Haarkreise die verschiedenen Variationen des Dickendurchmessers vertreten. Den Haarausfall habe ich nur an drei Tagen gezählt, er ergab am 3. 4. und 5. October 1859 die Zahlen 204, 282, 213. Das Verbältniss der Spitzenhaare zum Gesammtausfall war 1 zu 3,706. Jedes neunte Haar war ein Lanugahaar.

In diesem Stadium zeigte der Haarausfall alle wesentlichen Symptome der Alopecia pityrodes, zumal eine mässige Pityriasis capitis sich einstellte. Nur die straffere Anheftung der Cutis fehlte und ich bemerke hierbei, dass ich in keinem Stadium des Verlaufs dieses Falles die von einzelnen Autoren bei Rheumatismus der Kopfschwarte beschriebene Anschwellung der Cutis bemerkt habe.

Die abnorme Haarbildung äherdauerte die Schmerzhaftigkeit der Kopfsehwarte um einige Monate; am frühesten trat die normale Haarbildung in der Nähe der Stirn ein, am spätesten auf der Höhe des Scheitels; hier erfolgte auch keine vollständ̉ige Restitutio in integrum, es blieben vielmetr eine nicht unbeträchtliche Anzahl Wollhaare persistent. Immerhin erschien das Haar auch an dieser Stelle noch dicht. Im December 1866 erschien der Haarwuchs unverändert gut, bis auf jene kleine Stelle auf der Höhe des Scheitels, wo sich meist Wollhaar vorfand.

Die beiden anderen Fälle betrafen ein Mädchen von (beim Beginn der Krankheit) 21 und eine Frau von (beim Beginn der Krankheit) 32 Jahren; vager Muskel-Rheumatismus war bei Beiden längere Zeit vorausgegangen, dann folgten andauernde Kopfschmerzen, Gefühl von Hitze, ohne Anschwellung und obne Böthung der Haut. Das Haar ging auf dem ganzen Mittelkopf allmählich aus. Nach zwei, im zweiten Fall nach drei Jahren hörten die Schmerzen auf, 5 resp. 8 Monate darauf war der Kopf mit normal dicken Haaren gleichmässig bedeckt. Zwei Jahre später zẹigte der Nachwuchs eine normale Persistenz. 
Da das wesentlichste diagnostische Moment für die Annahme der rheumatischen Natur des Leidens der Kopfschmerz war, ist es nothwendig zu bemerken, dass intercurrente Kopfschmerzen bei der Alopecia pityrodes sehr häufig vorkommen. Besonders klagen die Kranken über einen Schmerz auf der Höhe des Scheitels, der mit einem Gefühl erhöhter Wärme verbunden ist und Stunden, wohl auch Tage lang andauert. Allein nie ist der Schmerz über den ganzen Mitelkopf gleichmässig verbreitet und niemals wird er durch Druck gesteigert, es treten stets Intermissionen ein, die Wochen und selbst Monate andauern.

Die letzte Krankheit, welche diagnostisch zu Irrthum Veranlassung geben kann, ist die Alopecia syphilitica. Die Veränderungen, welche bei Syphilitischen am Kopfhaar oft eintreten, beginnen in der Regel einige Wochen nach dern Entslehen der Haut-Syphiliden. In der Hälfte der Fälle entsteht ein Defluvium capillorum, in wenigen Wochen ist der Haarboden gleichmässig verdünnt, zuweilen völlig kahl. In der anderen Hälfte der Fälle erfolgt das Ausfallen nicht gleichmässig, sonderł entweder in ganz kleinen Gruppen oder selbst so, dass aus jedem Haarkreise ein oder zwei Haare ausfallen. In diesem Falle sind oft geröthete oder gelblich-braune Flecke, zuweilen Papeln und Pusteln vorhanden, oft aber zeigt die Cutis des Kopfes keine weiteren Symptome der Lues, nur Pityriasis capitis ist bemerkbar.

Mit dem Schwinden der anderweitigen Symptome der Syphilis tritt nun entweder normaler Haarwuchs ein, oder es bildet sich vollstăndige Kahlheit aus, oder die Haarkrankheit dauert Monate oder Jahre fort, um schliesslich doch noch in Genesung uberzugehen oder zu einer Calvities praematura zu führen.

Diese letzten Fälle können diagnostisch schwierig sein. Das Bild, welches der K!anke dem Arzte bei der ersten Untersuchung darbietet, gleicht fast völlig dem der Alopecia pityrodes; es fuhlt nur die straffere Anbeftung der Cutis. In den drei Fällen, welche ich sah und von denen zwei in Genesung übergingen, konnte die Diagnose auch abgesehen von dem Feblen der straffen Anheftung der Cutis mit Sicherheit gestellt werden, da der Krankheitsprozess sich nicht auf den Mittelkopf beschränkt, sondern auch den Hinterkopf und die Schläfen (allerdings in geringerem Grade) ergriffen hatte. 
Die möglichst scharfe Unterscheidung der beiden zuletzt angeführten Krankheitszustände von der Alopecia pilyrodes wird die Grenze der Therapie der Haarkrankheiten künftighin schärfer zeichnen und der überwuchernden Einmischung Unberufener allmählich entgegen wirken.

Aetiologie der Alopecia pityrodes.

Ein gewisses Alter, Erblichkeit, liederlicher Lebenswandel, kummervolles Leben und chronische Krankheiten haben von jeher für die Ursachen vorzeitiger Kahlheit gegolten. Der Begriff der chronischen Krankheit wurde in der Regel etwas weit aufgefasst, von einigen Autoren wurde sogar "behagliches Wohlleben " ("daher die Kahlköpfigkeit der Leute von gutem Embonpoint") dahin gerechnet.

Dass man sich an so wenig scbarfen oder engen Begriffen genügen liess, hat der Erkenntniss der ätiologischen Momente noch weniger Eintrag gethan als die Thatsache, dass die Autoren Alopecia pityrodes von den übrigen Arten der vorzeitigen Kahlheit, namentlich von der Calvities praematura nicht genügend unterschieden.

Ich habe 121 Fälle von Alopecia pityrodes untersucht, resp. behandelt, darunter jedoch nur 47 Fälle, je zwei Jahre lang oder länger beobachtet; nur diese letzteren sind für eine Erörterung der ätiologischen Verhältnisse brauchbar, weil ich nur an. ihnen die Vita anteacta wiederholt eruiren konnte.

Die 47 Krankheitsfälle zerfallen in zwei Gruppen:

1. Individuen, die abgesehen von der Haarkrankheit alle Zeichen einer kräftigen Gesundheit und einer guten Constitution hatten. (35 Fälle).

2. Individuen, bei welchen gleichzeitig mit dem Entstehen des Haarleidens eine Reihe von anderen Erscheinungen auftraten, welche die Annahme einer Störung der ganzen Constilution nahe legten, (ohne dass jedoch eine Constitutions-Krankheit auftrat). Diese Erscheinungen waren: Caries der Zähne, welche Anfangs die Zähne des Oberkiefers, später die des Unterkiefers ergriff und im Zeitraum von 5 bis 8 Jahren die Hälfte der Zähne oder einen noch grösseren Theil derselben zerstörte; chronischer Katarrh des Pharynx; geringe Neigung zur Heilung per primam intentionem bei Verletzung der Haut; gesteigerte Reizbarkeit der Nerven gegen psychische und physische Eindrücke; endlich schweissige Füsse. Alle diese 
Erscheinungen zusammen habe ich bei zwölf Kranken gefunden. (8 Männer, 4 Frauen).

Unter diesen zwölf Fällen liess sich zweimal (beides Männer) eine Erblichkeits-Anlage für die Alopecie nachweisen (einmal der Vater und zwei von den fünf Geschwistern sehr früh kahl; einmal Mutter und eine Schwester, der Vater und zwei Brüder nicht). Diese beiden Kranken und 8 von den übrigen 10 hatten aber in früheren Jahren (zwischen ihrem siebenten und dreizehnten Lebensjahr) an Impetigo capitis gelitten; die meisten angeblich mehrere Jahre nacheinander und jedesmal mehrere Monate lang. - Nach der Pubertät war der Kopfausschlag nicht wiedergekehrt.

Unter der ersten Gruppe von 35 Individuen (11 Fraven, 24 Männer) war 14 Mal (5 Frauen, 9 Männer) Erblichkeit nachweisbar. Unter diesen hatten zwei (beides Männer) in dem Alter von 8 bis 13 Jahren wiederholt an Eczemá oder Impetigo capitis gelitten. Unter den. übrigen 21 Fällen war 8 Mal (5 Männer, 3 Frauen) ein chronischer Hautausschlag des Kopies in den der Pubertäts-Entwickelung vorausgegangenen Jahren rorhanden gewesen, der bei 5 dieser Kranken (4 Mănner, 1 Frau) zu wiederholten Malen wiedergekehrt war.

Die 47 Patienten gehörten zu dem bei weitem grössten Theil (36) den vermögenden Stäuden an.

Die Schuppung der Kopfhaut war bei der grössten Hälfte der Kranken kurze Zeit oder einige Jahre nach Eintritt des Pubertät aufgetreten; in der kleineren Hälfte in der Mitte der zwanziger Jahre.

Bei der ersten Gruppe der Kranken war das Vorwiegen einer bestimmten Constitution nicht bemerkbar; gesund erschienen alle; Männer von hervorstechender solider und unsolider Lebensweise waren gleichmässig vertreten.

Ich konnte daher für die Alopecia pityrodes nur zwei prädisponirende Momente auffinden: die Erblichkeit und ein chronischer eczematöser oder impetiginöser KoplhautAusschlag in den dem Eintritt der Pubertät vorausgegangenen Jahren. Letzteres Leiden hatte bei fast allen denjenigen Kranken staitgefunden, bei welchen zugleich eine Reihe von anderen Symptomen bemerkt wurden, die man als Zeichen einer schwachen Constitution auffassen darf. Es ist diess dieselbe Symptomen-Gruppe, welche man in grösserer Intensităt bei tabes- 
cirenden Individuen wahrnimmt. Die Aehnlichkeit des Verjaufs und der Complicationen mancher Arten von Alopecie mit den Erscheinungen und begleitenden Symptomen der Alopecia tabescentium ist schon den Autoren, welche zuerst ubber diese Krankbeit schrieben, aufgefallen.

Vor der Pubertät habe ich, obwohl ich nun schon seit 8 Jahren auf die Krankheit achte, selbst das in dem früheren Aufsalz beschriebene erste Stadium des Leidens nie auftreten sehen, dass je näher dieser Epoche der Beginn der Krankbeit lag, desto rascher das erste Stadium in das zweite überging, habe ich bereits erwâhnt.

\section{XVIII.}

\section{Zur Lelire von der Tabes dorsualis.}

Von Dr. E. Cyon aus Petersburg.

Beinahe ein Jahr ist verflossen, seitdem meine Abhandlung über Tabes dorsualis veröffentlicht wurde. Die Kritik hat sich fast einstimmig zu Gunsten der in meiner Arbeit vertretenen Ansichten ausgesprochen. Von den bedeutendsten deutschen Klinikern wurde mir theils persönlich, theils durch Andere die schmeichelhafteste Anerkennung zu Theil und zwar hauptsächlich für meine Zurückweisung der Leyden'schen Schrift. Bekanntlich hat diese Schrift durch ihre Mängel meiner Kritik den reichsten Stoff dargeboten. Herr Leyden hat bis vor Kurzem die ihm ertheilte Zurechtweisung unbeantwortet gelassen. Nachdem sämmtliche Kritiker meiner Schrift sich mit dieser Zurechtweisung in Uebereinstimmung erklärt, war natürlich das Schweigen des Herrn Leyden nur so zu deuten, dass derselbe es vorgezogen habe, ein stilles "Pater peccavi" zu sagen, als durch eine Erwiderung sich der Gefahr auszusetzen, die begangenen Fehlgriffe dem lesenden Publicum von Neuem in's Gedächtniss zu bringen. Erst nacbdem die zwölfte Stunde schon längst geschlagen, hat er sich endlich doch $z u$ einer Antwort entschlossen, die im Arehiy f. pathol. Anat. Bd. XLI. Hft. 3 u. 4. 